\title{
Densité des vecteurs propres généralisés d'une classe d'opérateurs compacts non auto-adjoints et applications
}

\author{
Marie Thérèse Aimar ${ }^{1}$, Abdelkader Intissar ${ }^{2}$ et Jean Martin Paoli ${ }^{2}$ \\ 1 Département de Mathématiques, Université de Provence, Place V. Hugo, \\ F-13331 Marseille Cedex 3, France \\ 2 Département de Mathématiques, Université de Corte, Quartier Grossetti, F-20250 Corte, France
}

Received September 7, 1992; in revised form December 21, 1992

\begin{abstract}
We consider a closed densely defined linear operator $T$ in a Hilbert space $E$, and assume the existence of $\xi_{0} \in \varrho(T)$ such that $K=\left(T-\xi_{0} I\right)^{-1}$ is compact and the existence of $p>0$ such that $s_{n}(K)=\mathbf{o}\left(\left(n^{-1 / p}\right)\right)$, where $s_{n}(K)$ denotes the sequence of non-zero eigenvalues of the compact hermitian operator $\sqrt{K^{*} K}$. In this work, sufficient conditions (announced in [1]) are introduced to assure that the closed subspace of $E$ spanned by the generalized eigenvectors of $T$ coincides with $E$. These conditions are in particular verified by a family of non-self-adjoint operators arising in reggeon's field theory.
\end{abstract}

\section{Introduction}

Soit $T$ un opérateur linéaire fermé de domaine $D(T)$ dense dans un espace de Hilbert $E$ muni d'un produit scalaire noté $\langle$,$\rangle . On suppose que T$ est un opérateur à résolvante compacte et on désigne par $\varrho(T)$ son ensemble résolvant, alors son spectre $\sigma(T)=\mathbb{C}-\varrho(T)$ est discret, formé uniquement de valeurs propres et composé d'une suite de nombres complexes $\left\{\lambda_{\imath}\right\} i=1,2, \ldots$ qu'on ordonne en général de façon croissante tels que $\lim \left|\lambda_{i}\right|=+\infty$ lorsque $i \rightarrow+\infty$.

Rappel de quelques propriétés classiques [4] ou [12]. Soit $\lambda \in \mathbb{C}$, si on pose:

$-\operatorname{Ker}(\lambda I-T)^{k}=\left\{\varphi \in D\left(T^{k}\right) ;(\lambda I-T)^{k} \varphi=0\right\}$

et $\operatorname{Im}(\lambda I-T)^{k}=\left\{(\lambda I-T)^{k} \varphi ; \varphi \in D\left(T^{k}\right)\right\}$

$-\operatorname{Ker}\left(\lambda I-T^{*}\right)^{k}=\left\{\varphi \in D\left(T^{* k}\right) ;\left(\lambda I-T^{*}\right)^{k} \varphi=0\right\}$

et $\operatorname{Im}\left(\lambda I-T^{*}\right)^{k}=\left\{\left(\lambda I-T^{*}\right)^{k} \varphi ; \varphi \in D\left(T^{* k}\right)\right\}$,

alors on a:

1) Les sous espaces $\operatorname{Ker}(\lambda I-T)^{k}$ et $\operatorname{Im}(\lambda I-T)^{k}$ sont invariants par rapport à $T$.

2) $\operatorname{Ker}(\lambda I-T)^{k}$ est de dimension finie et $\operatorname{Im}(\lambda I-T)^{k}$ est fermé.

3) Il existe un entier $m$ tel que:

a) $\operatorname{Ker}(\lambda I-T)^{k} \subset \operatorname{Ker}(\lambda I-T)^{k+1}, 0 \leq k<m$ et $\operatorname{Ker}(\lambda I-T)^{k}=\operatorname{Ker}(\lambda I-T)^{k+1}$; $k \geq m$. 
b) $\operatorname{Im}(\lambda I-T)^{k+1} \subset \operatorname{Im}(\lambda I-T)^{k}, 0 \leq k<m$ et $\operatorname{Im}(\lambda I-T)^{k}=\operatorname{Im}(\lambda I-T)^{k+1}$; $k \geq m$.

c) $\operatorname{Ker}(\lambda I-T)^{m} \cap \operatorname{Im}(\lambda I-T)^{m}=\{0\}$ et $E=\operatorname{Ker}(\lambda I-T)^{m}+\operatorname{Im}(\lambda I-T)^{m}$ (Somme topologique).

d) $\left.(\lambda I-T)^{m}\right|_{D\left(T^{m}\right) \cap \operatorname{Im}(\lambda I-T)^{m}}$ est inversible.

e) $\operatorname{Im}\left(\bar{\lambda} I-T^{*}\right)^{k}=\left[\operatorname{Ker}(\lambda I-T)^{k}\right]^{\perp}$ et $\operatorname{Im}(\lambda I-T)^{k}=\left[\operatorname{Ker}\left(\bar{\lambda} I-T^{*}\right)^{k}\right]^{\perp}$.

$m$ est appelé indice de $\lambda$, la dimension de $\operatorname{Ker}(\lambda I-T)$ est la multiplicité géométrique de $\lambda$ et la dimension de $\left[\operatorname{Ker}(\lambda I-T)^{m}\right]$ est la multiplicité algébrique de $\lambda$.

Les éléments de $\left[\operatorname{Ker}(\lambda I-T)^{m}\right]$ sont appelés les vecteurs propres généralisés de $T$ associés à $\lambda$.

Afin de simplifier les notations, dans toute la suite on suppose $m=1$.

Soient $\lambda_{1}, \lambda_{2}, \ldots, \lambda_{n}, \ldots$ les valeurs propres de $T$, on a:

$$
\begin{aligned}
E= & \operatorname{Ker}\left(\lambda_{1} I-T\right)+\operatorname{Im}\left(\lambda_{1} I-T\right) \quad \text { (Somme topologique) } \\
E= & \operatorname{Ker}\left(\lambda_{2} I-T\right)+\operatorname{Im}\left(\lambda_{2} I-T\right) \quad \text { (Somme topologique) } \\
E= & \operatorname{Ker}\left(\lambda_{1} I-T\right)+\operatorname{Ker}\left(\lambda_{2} I-T\right) \\
& +\operatorname{Im}\left(\lambda_{1} I-T\right) \cap \operatorname{Im}\left(\lambda_{2} I-T\right) \quad \text { (Sommes topologiques) }
\end{aligned}
$$

Pour les $n$ premières valeurs propres on a:

$$
\begin{array}{rlr}
E= & \operatorname{Ker}\left(\lambda_{1} I-T\right)+\cdots+\operatorname{Ker}\left(\lambda_{n} I-T\right) & \\
& +\bigcap_{i=1}^{n} \operatorname{Im}\left(\lambda_{i} I-T\right) & \text { (Sommes topologiques) } \\
E= & \operatorname{Ker}\left(\bar{\lambda}_{1} I-T^{*}\right)+\cdots+\operatorname{Ker}\left(\bar{\lambda}_{n} I-T^{*}\right) \\
& +\bigcap_{i=1}^{n} \operatorname{Im}\left(\bar{\lambda}_{i} I-T^{*}\right) & \text { (Sommes topologiques) }
\end{array}
$$

Posons $M_{n}=\bigcap_{i=1}^{n} \operatorname{Im}\left(\lambda_{i} I-T\right)$ et $M_{n}^{*}=\bigcap_{i=1}^{n} \operatorname{Im}\left(\bar{\lambda}_{i} I-T^{*}\right)$, il est alors naturel de poser:

$$
M_{\infty}=\bigcap_{i=1}^{\infty} \operatorname{Im}\left(\lambda_{i} I-T\right) \quad \text { et } \quad M_{\infty}^{*}=\bigcap_{i=1}^{\infty} \operatorname{Im}\left(\bar{\lambda}_{i} I-T^{*}\right)
$$

Soient $D$ le plus petit sous espace vectoriel fermé contenant $\bigcup_{i=1}^{\infty} \operatorname{Ker}\left(\lambda_{i} I-T\right), D^{*}$ le plus petit sous espace vectoriel fermé contenant $\bigcup_{i=1}^{\infty} \operatorname{Ker}\left(\bar{\lambda}_{i} I-T^{*}\right)$ et $T_{\lambda}=\lambda I-T$. On commence par rappeler quelques propriétés classiques de l'opérateur $T_{\lambda}=\lambda I-T$ :

1) $\forall \lambda \in \mathbb{C}, \forall i \in \mathbb{N}$, on a: $T_{\lambda}\left(\operatorname{Ker}\left(\lambda_{i} I-T\right)\right) \subset \operatorname{Ker}\left(\lambda_{\imath} I-T\right)$.

2) $\forall \lambda \in \mathbb{C}, \forall i \in \mathbb{N}$, on a: $T_{\lambda}\left(D(T) \cap \operatorname{Im}\left(\lambda_{i} I-T\right)\right) \subset \operatorname{Im}\left(\lambda_{i} I-T\right)$.

3) $\forall \lambda \in \varrho(T), \forall i \in \mathbb{N}$, on a: $(\lambda I-T)^{-1}\left(\operatorname{Im}\left(\lambda_{i} I-T\right)\right) \subset\left(D(T) \cap \operatorname{Im}\left(\lambda_{i} I-T\right)\right)$

4) $\forall i \in \mathbb{N}, T_{\lambda_{2}}$ envoie bijectivement $D(T) \cap \operatorname{Im}\left(\lambda_{i} I-T\right) \operatorname{sur} \operatorname{Im}\left(\lambda_{i} I-T\right)$

5) $\forall(i, j) \in \mathbb{N}^{2} \times \mathbb{N}$ avec $i \neq j$, l'opérateur $T_{\lambda_{i}}$ envoie bijectivement $D(T) \cap \operatorname{Im}\left(\lambda_{i} I-T\right) \cap \operatorname{Im}\left(\lambda_{\jmath} I-T\right)$ sur $\operatorname{Im}\left(\lambda_{i} I-T\right) \cap \operatorname{Im}\left(\lambda_{j} I-T\right)$. 
Dans Dunford-Schwartz [4], on peut trouver aussi les résultats suivants:

1) $E=D+M_{\infty}^{*}=D^{*}+M_{\infty}$ (sommes directes orthogonales)

2) $\forall \lambda \in \mathbb{C}$, l'opérateur $T_{\lambda}=\lambda I-T$ est bijectif de $M_{\infty} \cap D(T)$ dans $M_{\infty}$.

3) $\forall \lambda \in \mathbb{C}$, l'opérateur $\left.(\lambda I-T)^{-1}\right|_{M_{\infty}}$ est quasi-nilpotent.

Remarque 1 .

1) $M_{\infty}$ est soit réduit au singleton $\{0\}$ soit de dimension infinie.

2) Le système des vecteurs propres généralisés de $T$ est dense dans $E$ si et seulement si $M_{\infty}^{*}=\{0\}$.

Proposition 1. L'application $\lambda \rightarrow f(\lambda)=\left\langle(\lambda I-T)^{-1} \varphi, \psi\right\rangle, \varphi \in M_{\infty}$ et $\psi \in E$ est analytique sur tout le champ complexe.

\section{Démonstration}

- Pour $\lambda_{0} \in \varrho(T)$, l'analyticité de la fonction $f(\lambda)=\left\langle(\lambda I-T)^{-1} \varphi, \psi\right\rangle$ au voisinage de $\lambda_{0}$ se déduit de l'analyticité de la résolvante de l'opérateur $T$.

- Pour $\lambda_{0} \in \sigma(T)$ d'indice $n_{0}$, il existe $\alpha_{0}>0$ tel que $\left\{\lambda,\left|\lambda-\lambda_{0}\right|<\alpha_{0}\right\}$ soit dans $\varrho(T)$ et on a le développement de Laurent:

$$
f(\lambda)=\left\langle(\lambda I-T)^{-1} \varphi, \psi\right\rangle=\sum_{k=0}^{\infty}\left(\lambda-\lambda_{0}\right)^{k}\left\langle A_{k} \varphi, \psi\right\rangle+\sum_{k=1}^{n_{0}} \frac{\left\langle B_{k} \varphi, \psi\right\rangle}{\left(\lambda-\lambda_{0}\right)^{k}},
$$

où $B_{1}$ est la projection sur $\operatorname{Ker}\left(\lambda_{0} I-T\right)$ parallèlement à $\operatorname{Im}\left(\lambda_{0} I-T\right)$ et où les autres coefficients de la série de Laurent vérifient:

- $\left(\lambda_{0} I-T\right) A_{0}=I-B_{1}$,

- $(-1)^{k}\left(\lambda_{0} I-T\right)^{k} A_{k}=A_{0}$ pour tout $k \geq 0$,

- $(-1)^{k}\left(\lambda_{0} I-T\right)^{k} B_{1}=B_{k+1}$ pour tout $0 \leq k \leq n_{0}-1$.

Comme $\operatorname{Ker} B_{1}=\operatorname{Im}\left(\lambda_{0} I-T\right)$, alors si $\varphi \in M_{\infty}, \varphi$ est dans $\operatorname{Im}\left(\lambda_{0} I-T\right)$ et donc dans $\operatorname{Ker} B_{1}$, d'où $B_{\imath} \varphi=0$ et par suite $B_{k+1} \varphi=0$ pour tout $0 \leq k \leq n_{0}-1$. $f$ est donc analytique en tout point au voisinage de $\lambda_{0}$. Il nous reste à montrer que $f$ est analytique au point $\lambda_{0}$. Pour cela, on va montrer que $f\left(\lambda_{0}\right)=\left\langle A_{0} \varphi, \psi\right\rangle$.

Comme $\left(\lambda_{0} I-T\right) A_{0}=I-B_{1}, A_{0} \varphi \in D(T)$ et puisque $(-1)^{k}\left(\lambda_{0} I-T\right)^{k} A_{k}=A_{0}$ pour tout $k \geq 0$, alors $A_{k} \varphi \in D\left(T^{k}\right)$ et $A_{0} \varphi \in \operatorname{Im}\left(\lambda_{0} I-T\right)^{k}$. Par conséquent $A_{0} \varphi \in \operatorname{Im}\left(\lambda_{0} I-T\right)$ et il en résulte que $A_{0} \varphi \in D(T) \cap \operatorname{Im}\left(\lambda_{0} I-T\right)$. De plus $\left(\lambda_{0} I-T\right) A_{0} \varphi=\left(I-B_{1}\right) \varphi=\varphi$ puisque $\varphi \in M_{\infty} \subset \operatorname{Ker}\left(B_{1}\right)$ et $\left(\lambda_{0} I-T\right)\left[\left(\lambda_{0} I-\right.\right.$ $\left.T)\left.\right|_{M_{\infty}}\right]^{-1} \varphi=\varphi$ avec $\left[\left.\left(\lambda_{0} I-T\right)\right|_{M_{\infty}}\right]^{-1} \varphi \in D(T) \cap \operatorname{Im}\left(\lambda_{0} I-T\right)$.

Par suite on en déduit que $A_{0} \varphi=\left[\left.\left(\lambda_{0} I-T\right)\right|_{M_{\infty}}\right]^{-1} \varphi$ et donc

$$
\left\langle A_{0} \varphi, \psi\right\rangle=\left\langle\left[\left.\left(\lambda_{0} I-T\right)\right|_{M_{\infty}}\right]^{-1} \varphi, \psi\right\rangle=f\left(\lambda_{0}\right) .
$$

\section{Quelques théorèmes sur la densité des vecteurs propres généralisés}

Soit $E$ un espace de Hilbert sur $\mathbb{C}$ muni du produit scalaire $\langle.,$.$\rangle et de la norme$ associée $\|$. $\|$ et $T$ un opérateur linéaire fermé de domaine $D(T)$ dense dans $E$.

On rappelle qu'un opérateur compact $K$ appartient à la classe $\ell_{p}$ de Carleman si la série $\sum_{n \geq 1}^{\infty}\left[s_{n}\left(\sqrt{K^{*} K}\right)\right]^{p}$ converge où $s_{n}\left(\sqrt{K^{*} K}\right), n=1,2, \ldots$ désigne la suite des valeurs propres de l'opérateur compact hermitien positif $\sqrt{K^{*} K}$. 
Dans [10], Lang et Locker ont utilisé l'inégalité de Carleman et le principe de Phragmén-Lindelöf pour établir des conditions suffisantes qui garantissent la densité des vecteurs propres généralisés d'un opérateur à résolvante de Hilbert-Schmidt; leurs conditions généralisent certaines conditions données par Dunford et Schwartz dans [4] (corollaire 31, Ch XI, p: 1042). Une version de leurs résultats est la suivante:

Théoréme 1 (Lang et Locker [10]). Soit $T$ un opérateur linéaire fermé de domaine $D(T)$ dense dans un Hilbert $E$; on suppose qu'il existe $\xi_{0} \in \varrho(T)$ tel que $R_{\xi_{0}}(T)=\left(\xi_{0} I-T\right)^{-1}$ soit un opérateur de Hilbert-Schmidt. Soient cinq demi-droites $\gamma_{0}, \gamma_{1}, \gamma_{2}, \gamma_{3}, \gamma_{4}$ du plan complexe issues de l'origine telles que tout angle formé par deux demi-droites adjacentes soit plus petit que $\pi / 2$. On suppose que pour $|\lambda|$ assez grand, la résolvante $R_{\lambda}(T)$ est bornée sur $\gamma_{0}, \gamma_{1}, \gamma_{2}, \gamma_{3}, \gamma_{4}$ et que sur l'une de ces demi-droites elle satisfait l'inégalité: $\left\|R_{\lambda}(T)\right\|=O(1 /|\lambda|)$ lorsque $|\lambda| \rightarrow \infty$. Alors l'espace engendré par les vecteurs propres généralisés de $T$ est dense dans $E$.

En utilisant un théorème de Macaev [6] et le principe de Phragmén-Lindelöf [13], nous proposons dans ce travail de généraliser le théorème précédent à un opérateur $T$ à résolvante $K=\left(T-\xi_{0} I\right)^{-1}$ compacte pour lequel il existe $p>0$ tel que $s_{n}(K)=\mathbf{o}\left(\left(n^{-1 / p}\right)\right),(n \rightarrow \infty)$.

Remarque 2. Il est clair que si un opérateur compact $K$ appartient à la classe $\ell_{p}$ de Carleman alors $s_{n}(K)=\mathbf{o}\left(n^{-1 / p}\right),(n \rightarrow \infty)$.

Théorème 2. Soit $T$ un opérateur linéaire fermé de domaine $D(T)$ dense dans un Hilbert E. On suppose:

$H_{1}$ ) Il existe $\xi_{0} \in \varrho(T)$ tel que $K=\left(T-\xi_{0} I\right)^{-1}$ soit un opérateur compact.

$H_{2}$ ) Il existe $p>0$ tel que $s_{n}(K)=\mathbf{o}\left(n^{-1 / p}\right)(n \rightarrow \infty)$.

$\left.H_{3}\right)$ Pour $|\lambda|$ assez grand, la résolvante $R_{\lambda}(T)=(T-\lambda I)^{-1}$ est bornée sur $m$ demidroites $\gamma_{1}, \gamma_{2}, \ldots, \gamma_{m}$ issues de l'origine divisant tout le plan complexe et telles que l'angle formé par deux demi-droites adjacentes quelconques soit strictement plus petit que $\pi / p(m \geq[2 p]+1$ où $[2 p]$ désigne la partie entière de $2 p)$.

$H_{4}$ ) Il existe une suite $\xi_{n} \in \varrho(T)$ telle que $R_{\xi_{n}}(T)$ converge faiblement vers zéro lorsque $\left|\xi_{n}\right|$ tend vers l'infini.

Alors le système des vecteurs propres généralisés de T est dense dans $E$.

Proposition 2. 1) Si un opérateur T vérifie les hypothèses du Théorème 2 , alors il en est de même pour son adjoint $T^{*}$.

2) On pose $M_{\infty}=\bigcap_{k=1}^{\infty} \operatorname{Im}\left(\lambda_{k} I-T\right)$ (fermé) où $\operatorname{Im}\left(\lambda_{k} I-T\right)$ désigne l'image de l'opérateur $\lambda_{k} I-T$ et $\left\{\lambda_{k}\right\} k=1,2, \ldots$ sont les valeurs propres de $T$. Si un opérateur $T$ vérifie les hypothèses du Théorème 2, alors l'espace engendré par les vecteurs propres généralisés de $T$ est dense dans $E$ si et seulement si $M_{\infty}=\{0\}$.

3) Si $K$ est opérateur compact sur un Hilbert $E$ et $F$ un sous espace de Hilbert stable par $K$ alors $s_{n}\left(\left.K\right|_{F}\right) \leq s_{n}(K)$ pour tout $n \geq 1$.

4) Sous les hypothèses $H_{1}$ et $H_{2}$ du Théorème 2 on a

$$
\left.\left.s_{n}(\lambda I-T)^{-1}\right|_{M_{\infty}}\right)=\mathbf{o}\left(n^{-1 / p}\right)(n \rightarrow \infty)
$$

Démonstration. 1) Si un opérateur $T$ vérifie les hypothèses du Théoréme 2 alors son adjoint $T^{*}$ les vérifie aussi puisque $\varrho\left(T^{*}\right)=\overline{\varrho(T)}$ et $\left(\bar{\lambda} I-T^{*}\right)^{-1}=\left[(\lambda I-T)^{-1}\right]^{*}$ pour tout $\lambda \in \varrho(T)$. En effet les vérifications des hypothèses $H_{1}$ et $H_{3}$ du Théorème 2 se font facilement; quant à celle de $H_{4}$, il suffit de remarquer que la convergence 
faible vers zéro de $R_{\xi_{n}}\left(T^{*}\right)$ lorsque $\left|\xi_{n}\right|$ tend vers l'infini est équivalente à celle de $R_{\bar{\xi}_{n}}(T)$. Le seul point un peu moins trivial est la vérification de l'hypothèse $H_{2}$ qui résulte de la propriété importante $s_{n}(K)=s_{n}\left(K^{*}\right)$; cette dernière se démontrant rapidement en utilisant le développement de Schmidt d'un opérateur compact.

2) Du fait que le système des vecteurs propres généralisés d'un opérateur $T$ est dense, il ne résulte pas en général que le système des vecteurs propres généralisés de $T^{*}$ soit dense, mais sous les hypothèses de notre théorème, il suffit de combiner le (1) de cette proposition et la caractérisation de la densité des vecteurs propres généralisés donnée dans la Remarque 1.

3) La propriété (3) de cette proposition est une conséquence du théorème d'Allahverdiev [6] rappelé ci-dessous:

Théorème 3 (Allahverdiev [6]). Si K est un opérateur compact sur un Hilbert $E$, pour tout $n \geq 1$ on a: $s_{n}(K)=d\left(K, K_{n-1}(E)\right)$ où $K_{n-1}(E)$ désigne le sous espace des opérateurs bornés $L(E)$, de rang $n-1$; la distance étant prise pour la norme de $L(E)$ (espace des opérateurs bornés).

Si $F$ est un sous espace de Hilbert de $E, L(F)$ s'injecte dans $L(E)$ de façon à avoir $d_{L(F)}\left(\left.K\right|_{F}, K_{n-1}(F)\right) \leq d_{L(E)}\left(K, K_{n-1}(E)\right)$ et donc $s_{n}\left(\left.K\right|_{F}\right) \leq s_{n}(K)$ pour tout $n \geq 1$.

4) Soit $\xi_{0} \in \varrho(T)$, comme $\left.\left(T-\xi_{0} I\right)^{-1}\right|_{M_{\infty}}=\left(\left.T\right|_{M_{\infty}}-\xi_{0} I\right)^{-1}$, on obtient en combinant l'hypothèse $H_{2}$ de notre théorème et la propriété (3) de cette proposition: $\left.s_{n}\left(\left.\left(T-\xi_{0} I\right)^{-1}\right|_{M_{\infty}}\right)=\mathbf{o}\left(n^{-1 / p}\right)\right)(n \rightarrow \infty)$.

Soit $\xi_{0} \in \varrho(T)$, on pose $K_{1}=\left.\left(T-\xi_{0} I\right)^{-1}\right|_{M_{\infty}}$ et soit $\lambda \in \mathbb{C}-\left\{\xi_{0}\right\}$ on a:

i) $\left.(\lambda I-T)\right|_{M_{\infty}}=\lambda I-\left.T\right|_{M_{\infty}}=\lambda I-\xi_{0} I+\xi_{0} I-\left.T\right|_{M_{\infty}}$.

ii) $\left.(\lambda I-T)\right|_{M_{\infty}} \cdot K_{1}=\left[\left(\lambda-\xi_{0}\right) I+\left(\xi_{0} I-\left.T\right|_{M_{\infty}}\right)\right] \cdot K_{1}^{\infty}=\left(\lambda-\xi_{0}\right) K_{1}+I$.

iii) $\left.\frac{1}{\xi_{0}-\lambda}(\lambda I-T)\right|_{M_{\infty}} \cdot K_{1}=-K_{1}+\frac{I}{\xi_{0}-\lambda}=\frac{I}{\xi_{0}-\lambda}-K_{1}$.

iv) $\left.\frac{1}{\xi_{0}-\lambda}(\lambda I-T)\right|_{M_{\infty}} \cdot K_{1}\left(\frac{I}{\xi_{0}-\lambda}-K_{1}\right)^{-1}=I \operatorname{sur} M_{\infty}$.

On montre de même que:

$$
\left.\frac{1}{\xi_{0}-\lambda} \cdot K_{1}\left(\frac{I}{\xi_{0}-\lambda}-K_{1}\right)^{-1}(\lambda I-T)\right|_{M_{\infty}}=I \operatorname{sur} D(T) \cap M_{\infty} .
$$

d'où l'on déduit que:

$\left(\left.(\lambda I-T)\right|_{M_{\infty}}\right)^{-1}=\frac{1}{\xi_{0}-\lambda} \cdot K_{1}\left(\frac{I}{\xi_{0}-\lambda}-K_{1}\right)^{-1}$ qui est fermé et partout défini sur $M_{\infty}$ donc borné (par le théorème du graphe fermé). Par suite, comme $s_{n}\left(B \cdot K_{1}\right) \leq$ $\|B\| \cdot s_{n}\left(K_{1}\right)$ pour tout opérateur $B$ borné, on a:

$$
\left.\left.s_{n}(\lambda I-T)^{-1}\right|_{M_{\infty}}\right)=\mathbf{o}\left(n^{-1 / p}\right)(n \rightarrow \infty)
$$

Démonstration du Théorème 2. La démonstration du Théorème 2 repose sur la proposition précédente et le théorème de Macaev [6] rappelé ci-dessous:

Théorème 4 (Macaev [6]). Soit $K$ un opérateur quasi-nilpotent, on note $M(r)$ $(0 \leq r<\infty)$ la fonction:

$$
M(r)=\underset{|\lambda|=r}{\operatorname{Max}}\left\|(I-\lambda K)^{-1}\right\|
$$


Si $s_{n}(K)=O\left(n^{-1 / p}\right)$, (respectivement $\mathbf{o}\left(n^{-1 / p}\right)$ ) pour un certain $p>0$, alors $\log M(r)=O\left(r^{p}\right)$ (respectivement $\mathbf{o}\left(r^{p}\right)$ ). Si $K \in \ell_{p}$, on a de plus: $\int_{0}^{\infty} \frac{\log M(r)}{r^{p+1}} d r<\infty$

En vertu de ce théorème et de l'hypothèse $H_{3}$ de notre théorème, la fonction $f(\lambda)=\left\langle(\lambda I-T)^{-1} \varphi, \psi\right\rangle$ où $\lambda \in \mathbb{C}, \varphi \in M_{\infty}$ et $\psi \in E$ vérifie les hypothèses du principe de Phragmén-Lindelöf [13], elle est donc bornée sur tous les secteurs limités par les demi-droites $\gamma_{1}, \gamma_{2}, \ldots, \gamma_{m}$, c'est à dire sur $\mathbb{C}$ tout entier; $f$ fonction entière bornée sur $\mathbb{C}$ est donc constante. D'autre part, de l'hypothèse $H_{4}$ de notre théorème, on déduit que $f$ est identiquement nulle. Ainsi, pour tout $\varphi \in M_{\infty}$ et $\psi \in E$, $\left\langle(\lambda I-T)^{-1} \varphi, \psi\right\rangle=0$ donc $(\lambda I-T)^{-1} \varphi=0$ et par injectivité de $\left(\lambda I-\left.T\right|_{M_{\infty}}\right)^{-1}$ on obtient $M_{\infty}=\{0\}$, ce qui achève la démonstration.

Remarque 3. Notre théorème généralise d'autres théorèmes établis sur la densité des vecteurs propres généralisés, présentés ci-dessous sous forme simplifiée:

Théorème 5 (Macaev [6]). Soit $T$ un opérateur linéaire fermé de domaine $D(T)$ dense dans un espace de Hilbert $E$ et $1 \leq p<\infty$ vérifiant:

i) Il existe $\xi_{0} \in \varrho(T)$ tel que l'opérateur $K=\left(T-\xi_{0} I\right)^{-1}$ soit compact, et tel que $s_{n}(K)=\mathbf{o}\left(n^{-1 / p}\right)$ lorsque $n$ tend vers l'infini.

ii) Il existe une constante $\varepsilon>0$ telle que l'image numérique de l'opérateur $T$ soit incluse dans le secteur $S_{p}=\left\{\lambda \in \mathbb{C} ;-\frac{\pi}{2 p}+\varepsilon \leq \operatorname{Arg}(\lambda) \leq \frac{\pi}{2 p}-\varepsilon\right\}$.

Alors l'espace engendré par les vecteurs propres généralisés de T est dense dans $E$.

L'ingrédient essentiel permettant de déduire ce théorème de notre théorème est le lemme suivant:

Lemme 1. Sous les hypothèses du théorème ci-dessus, sur toute demi-droite $\gamma d u$ plan complexe issue de l'origine et n'appartenant pas au secteur $S_{p}$, la norme de la résolvante $\left\|(T-\lambda I)^{-1}\right\|$ est bornée indépendamment de $\lambda$.

Démonstration. On suppose par exemple que $0 \in \varrho(T)$, on en déduit que $\left\langle T^{-1} \psi, \psi\right\rangle /\|\psi\|^{2} \in S_{p} \forall \psi \in E$. Soit $\lambda \in \gamma$ avec $|\lambda|$ assez grand et n'appartenant pas au secteur $S_{p}$, on a:

$$
\left|\frac{1}{\lambda}-\left\langle T^{-1} \psi, \psi\right\rangle /\|\psi\|^{2}\right| \geq\left|\sin \left(\theta_{0}\right)\right| /|\lambda|
$$

où $\theta_{0}$ désigne le plus petit des deux angles formés par la demi-droite $\gamma$ et les bords du secteur $S_{p}$. En simplifiant par $|\lambda|$ on obtient:

$$
\left|\left\langle\left(I-\lambda T^{-1}\right) \psi, \psi\right\rangle\right| \geq\|\psi\|^{2} \cdot\left|\sin \left(\theta_{0}\right)\right| .
$$

D'où on déduit (en appliquant l'inégalité de Cauchy) que:

$$
\left\|\left(I-\lambda T^{-1}\right)^{-1}\right\| \leq 1 /\left|\sin \left(\theta_{0}\right)\right| \text {. }
$$

Enfin, comme $(T-\lambda I)^{-1}=T^{-1}\left(I-\lambda T^{-1}\right)^{-1}$ on obtient

$$
\left\|(T-\lambda I)^{-1}\right\| \leq\left\|T^{-1}\right\| /\left|\sin \left(\theta_{0}\right)\right|,
$$

ce qui prouve que la résolvante est uniformément bornée sur $\gamma$.

Théorème 6 (Keldys [6]). Soit E un espace de Hilbert. 
1) Soient $H$ un opérateur auto-adjoint de classe $\ell_{p}$ de Carleman, $S$ un opérateur compact et $A=H(I+S)$. Si l'opérateur $A$ est injectif, alors le système de ses vecteurs propres généralisés est dense dans $E$.

2) Soit $A=L+T$, où $L$ est un opérateur auto-adjoint à spectre discret, c'est-àdire son spectre est formé de valeurs propres isolées de multiplicité finie avec un seul point d'accumulation à l' infini, et $T$ un opérateur L-compact. On suppose qu'il existe $\lambda_{0} \in \varrho(L)$ tel que l'opérateur $\left(L-\lambda_{0} I\right)^{-1} T\left(L-\lambda_{0} I\right)^{-1}$ soit de classe $\ell_{p}$ de Carleman. Alors le système des vecteurs propres généralisés de l'opérateur $A$ est dense dans $E$.

Théorème 7 (Reed et Simon [11]). 1) Soit A un opérateur à trace et strictement $m$ accrétif c'est-à-dire qu'il existe une constante $\varepsilon>0$ telle que l'image numérique de l'opérateur $A$ soit incluse dans le secteur $S_{1}=\left\{\lambda \in \mathbb{C},-\frac{\pi}{2}+\varepsilon \leq \operatorname{Arg}(\lambda) \leq \frac{\pi}{2}-\varepsilon\right\}$. Alors l'espace engendré par les vecteurs propres généralisés de A est dense dans E. 2) Soit A le générateur infínitésimal d'un semi-groupe holomorphe de contraction. On suppose que $(A+I)^{-1}$ est un opérateur à trace. Alors l'espace engendré par les vecteurs propres généralisés de $A$ est dense dans $E$.

\section{Application à l'opérateur de Gribov}

La théorie des champs de reggeons a été inventée par Gribov en 1967 [5] afin de décrire le comportement à haute énergie des sections efficaces de collisions de particules élémentaires. Elle est caractérisée par une famille d'opérateurs non autoadjoints s'exprimant en fonction des opérateurs de création et d'annihilation usuels.

A $\mathbf{n}$ sites et en une dimension transverse, la famille d'opérateurs de Gribov se présente sous la forme:

Soit

$$
H=\lambda^{\prime} \sum_{\jmath=1}^{n} S_{\jmath}+\mu \sum_{\jmath=1}^{n} H_{1 j}+\alpha \sum_{j=1}^{n-1} H_{12 \jmath}+i \lambda \sum_{j=1}^{n} H_{2 j}=\operatorname{Re} \cdot H+i \operatorname{Im} \cdot H ; \quad i^{2}=-1
$$

où

$$
\begin{aligned}
& -S_{j}=A_{j}^{* 2} A_{j}^{2} \\
& -H_{1 j}=A_{j}^{*} A_{j} \\
& -H_{12 \jmath}=A_{j+1}^{*} A_{j}+A_{\jmath}^{*} A_{j+1} \\
& -H_{2 \jmath}=A_{j}^{*}\left(A_{j}+A_{j}^{*}\right) A_{j}
\end{aligned}
$$

avec $\left(\lambda^{\prime}, \mu, \alpha, \lambda\right) \in \mathbb{R}^{4}$.

Cette famille d'opérateurs non auto-adjoints agit sur l'espace de Bargmann-Segal [3]:

$$
\begin{gathered}
E=\left\{\varphi: \mathbb{C}^{n} \rightarrow \mathbb{C} \text { analytiques; } \int_{\mathbb{C}^{n}} \operatorname{Exp}\left(-\sum_{j=1}^{n}\left|z_{j}\right|^{2}\right)|\varphi(z)|^{2} d z_{j} d \bar{z}_{j}<\infty\right. \\
\text { et } \varphi(0)=0\} .
\end{gathered}
$$

Dans cette représentation les opérateurs $A_{j}^{*}$ et $A_{j}$ ne sont autres que la multiplication par $z_{j}$ et la dérivation par rapport à $z_{j}$. 
$H$ s'écrit ainsi:

$H=\sum_{j=1}^{n}\left(\lambda^{\prime} z_{j}^{2}+i \lambda z_{\jmath}\right) \partial^{2} / \partial z_{j}^{2}+\left(i \lambda z_{j}^{2}+\mu z_{j}\right) \partial / \partial z_{j}+\alpha \sum_{j=1}^{n-1}\left(z_{j+1} \partial / \partial z_{\jmath}+z_{j} \partial / \partial z_{j+1}\right)$.

Pour cette famille d'opérateurs, Ando, Intissar et Zerner ont développé dans $[2,7,9]$ une théorie spectrale complète: Définition du domaine, construction du semigroupe associé, analyse spectrale et comportement asymptotique de ce semi-groupe, analyticité de la plus petite valeur propre de $H$ par rapport au paramètre $\mu$ (intercept de Pomeron) et développement en série entière par rapport au paramètre $\alpha$ (pente de la trajectoire de Regge).

Nous commençons par préciser quelques notations et donner sous forme d'une proposition quelques propriétés spectrales de l'opérateur $H$ étudiées dans [7].

- $P_{0}$ désigne l'ensemble des polynômes qui s'annulent à l'origine.

- $S=\sum_{j=1}^{n} S_{j}$ de domaine $D(S)=\{\varphi \in E ; S \varphi \in E\}$.

- le domaine maximal de $H$ est $D(H)=\{\varphi \in E ; H \varphi \in E\}$.

- Le domaine minimal de $H$ est:

$$
D_{\text {min }}(H)=\left\{\varphi \in E ; \exists p_{n} \in P_{0} \text { et } \exists \psi \in E ; p_{n} \rightarrow \varphi \text { et } H_{p_{n}} \rightarrow \psi\right\} \text {. }
$$

Alors on a:

Proposition 3. (Intissar [7]). i) Pour $\lambda^{\prime} \neq 0, D(H)=D_{\min }(H)=\bigcap_{j=1}^{n} D\left(A_{j}^{4}\right)=$
$D(S)$. ii) Pour $\lambda^{\prime} \neq 0$ il existe $\beta>0 ; H+\beta I$ soit bijectif.

Théorème 8. 1) Pour $\lambda^{\prime}>0, H$ est un opérateur à résolvante de classe $\ell_{p}(p>n / 2)$ de Carleman.

2) Pour $\lambda^{\prime}>0$ il existe $\beta_{0}>0$ tel que $H+\beta_{0} I$ soit accrétif et d'image numérique incluse dans un secteur d'ouverture $\pi / p$.

3) Pour $\lambda^{\prime}>0$, le sous espace engendré par les vecteurs propres généralisés de $H$ est dense dans l' espace de Bargmann-Segal.

Démonstration. 1) Soit $H_{1}=\mu \sum_{j=1}^{n} H_{1 j}+\alpha \sum_{j=1}^{n-1} H_{12 j}+i \lambda \sum_{j=1}^{n} H_{2 \jmath}$ donc

$$
H+\beta I=\lambda^{\prime} S+\beta I+H_{1}=\left(I+H_{1}\left(\lambda^{\prime} S+\beta I\right)^{-1}\right)\left(\lambda^{\prime} S+\beta I\right)
$$

d'où $(H+\beta I)^{-1}=\left(\lambda^{\prime} S+\beta I\right)^{-1}\left(I+H_{1}\left(\lambda^{\prime} S+\beta I\right)^{-1}\right)^{-1}$, l'opérateur $\left(\lambda^{\prime} S+\beta I\right)$ est auto-adjoint positif et ses valeurs propres sont

$$
\beta_{k_{1} k_{2} \ldots k_{n}}=\lambda^{\prime}\left(k_{1}\left(k_{1}-1\right)+\left(k_{2}\left(k_{2}-1\right)+\ldots+k_{n}\left(k_{n}-1\right)\right)+\beta .\right.
$$

Si $p \geq n / 2$ la série $\sum_{k_{1} \geq 1} \sum_{k_{2} \geq 1} \ldots \sum_{k_{n} \geq 1}\left(1 / \beta_{k_{1} k_{2} \ldots k_{n}}\right)^{p}$ est convergente. Comme $\left(\lambda^{\prime} S+\beta I\right)^{-1}$ appartient au moins à $\ell_{p}(p>n / 2)$ alors il en est de même pour $(H+\beta I)^{-1}$.

2) La démonstration de ces propriétés repose sur le lemme suivant [7, p. 267]

Lemme 2 (Intissar [7]). 1) Soit $j \in[1, n]$, et $k$ un entier non nul, alors pour tout $p$ polynôme en $A_{j}$ et $A_{j}^{*}$ de degré $r<2 k$ on $a: \forall \varepsilon>0, \exists C_{\varepsilon}>0$ tel que $\forall \varphi \in D\left(A_{j}^{2 k}\right) ;\left|\left\langle p\left(A_{j}, A_{j}^{*}\right) \varphi, \varphi\right\rangle\right| \leq \varepsilon\left\langle A_{j}^{* k} A_{j}^{k} \varphi, \varphi\right\rangle+C_{\varepsilon}\|\varphi\|^{2}$. 
2) Soit $j \in[1, n]$, pour tout entier $k$, on $a: \forall \varepsilon>0, \exists C_{\varepsilon}>0$ tel que $\forall \varphi \in D\left(A_{\jmath}^{k+1}\right)$; $\left\|A_{j}^{k} \varphi\right\|^{2} \leq \varepsilon\left\|A_{j}^{k+1} \varphi\right\|^{2}+C_{\varepsilon}\|\varphi\|^{2}$.

De ce lemme, on déduit que:

$\forall \varepsilon>0, \exists C_{\varepsilon}>0$ tel que $|\operatorname{Im}\langle H \varphi, \varphi\rangle| \leq|\lambda| \varepsilon\langle S \varphi, \varphi\rangle+|\lambda| C_{\varepsilon}\|\varphi\|^{2} \forall \varphi \in D(S)$ et donc $|\operatorname{Im}\langle H \varphi, \varphi\rangle| \leq\left|\frac{\lambda}{\lambda^{\prime}}\right| \varepsilon\left\langle\lambda^{\prime} S \varphi, \varphi\right\rangle+|\lambda| C_{\varepsilon}\|\varphi\|^{2} \forall \varphi \in D(S)$.

Comme $\alpha\left\langle\sum_{\jmath=1}^{n-1}\left(A_{j+1}^{*} A_{\jmath}+A_{\jmath}^{*} A_{\jmath+1}\right) \varphi, \varphi\right\rangle \geq-2|\alpha| \sum_{\jmath=1}^{n}\left\|A_{\jmath} \varphi\right\|^{2}$, il en résulte que $\operatorname{Re}\langle H \varphi, \varphi\rangle \geq\left\langle\lambda^{\prime} S \varphi, \varphi\right\rangle-(|\mu|+2|\alpha|) \sum_{j=1}^{n}\left\langle A_{\jmath}^{*} A_{\jmath} \varphi, \varphi\right\rangle$.

En appliquant à nouveau le lemme précédent $\left\langle A_{j}^{*} A_{\jmath} \varphi, \varphi\right\rangle ; 1 \leq j \leq n$, on obtient:

$$
\begin{aligned}
\forall \delta>0, \exists C_{\delta}>0 ; \operatorname{Re}\langle H \varphi, \varphi\rangle \geq & \left\langle\lambda^{\prime} S \varphi, \varphi\right\rangle-(|\mu|+2|\alpha|)\left(\delta / \lambda^{\prime}\right)\left\langle\lambda^{\prime} S \varphi, \varphi\right\rangle \\
& -(|\mu|+2|\alpha|) C_{\delta}\|\varphi\|^{2} .
\end{aligned}
$$

En choisissant $\delta \leq \lambda^{\prime} /(|\mu|+2|\alpha|)$ et $\beta \geq(|\mu|+2|\alpha|) C_{\delta}$ on en déduit que $H+\beta I$ est un opérateur accrétif. Maintenant, si on choisit

$$
\varepsilon<\left(\lambda^{\prime} /|\lambda|\right) \operatorname{tg}(\pi / 2 p) \cdot\left(1-(|\mu|+2|\alpha|)\left(\delta / \lambda^{\prime}\right)\right)
$$

et $\beta_{0}>(|\mu|+2|\alpha|) C_{\delta}+\left(|\lambda| C_{\varepsilon} / \operatorname{tg}(\pi / 2 p)\right)$, on déduit que $H+\beta I$ est accrétif et que son image numérique est incluse dans le secteur d'ouverture $\pi / p$.

3) Il suffit d'appliquer le Théorème 2 ou le Théorème 5.

\section{Bibliographie}

1. Aimar, M.T., Intissar, A. et Paoli, J.M.: Densité des vecteurs propres généralisés d'une classe d'opérateurs compacts non auto-adjoints. C. R. Acad. Sci. Paris, 315, Série I (1992)

2. Ando, T. et Zerner, M.: Sur une valeur propre d'un opérateur, Commun. Math. Phys. 93 (1984)

3. Bargmann, V.: On a Hilbert space of analytic functions and an associated integral transform. I. Commun. Pure App. Math 14 (1962)

4. Dunford, N, Schwartz, J.T.: Linear operators. II, III. In: Pure Appl. Math., Vol 7. New York: Interscience, 1963

5. Gribov, V.: J.E.T.P (Sov. Phys), 26 (1968), 414

6. Gohberg, I.C., Krein, M.G.: Introduction to the theory of linear non-self-adjoint operators. A.M.S 18 (1969)

7. Intissar, A.: Etude spectrale d'une famille d'opérateurs non-symétriques intervenant dans la théorie des champs de reggeons. Commun. Math. Phys. 113 (1987)

8. Intissar, A.: Quelques nouvelles propriétés spectrales de l'hamiltonien de la théorie des champs de reggeons. C. R. Acad. Sci. Paris 308 Série I (1989)

9. Intissar, A.: Théorie spectrale dans l'espace de Bargmann. Cours de D.E.A, Université de Besançon (1989)

10. Lang, P., Locker, J.: Denseness of the generalized eigenvectors of an $H-S$ discrete operator. J. Funct. Anal. 82, No. 2 (1989)

11. Reed, M., Simon, B.: Methods of modern mathematical physics. IV. Analysis of operators. London, New York: Academic Press, 1978

12. Taylor, A.E., Lay, D.C.: Introduction to functional analysis. New York: Wiley, 1980

13. Titchmarsh, E.C.: The theory of functions. Oxford: Oxford University Press 
